\title{
Erratum: Hierarchy of Azimuthal Anisotropy Harmonics in Collisions of Small Systems from the Color Glass Condensate [Phys. Rev. Lett. 121, 052301 (2018)]
}

\author{
Mark Mace, Vladimir V. Skokov, Prithwish Tribedy, and Raju Venugopalan
}

(Received 13 June 2019; published 18 July 2019)

DOI: 10.1103/PhysRevLett.123.039901

In our Letter, we reported on calculations describing the hierarchy of azimuthal anisotropies in $p / d /{ }^{3} \mathrm{He}+\mathrm{Au}$ collisions at RHIC energies within the dilute-dense color glass condensate (CGC) framework. We have since discovered an error in the numerical code which produced the results presented in the Letter; a factor of $\hbar c$ was missing in converting units of momenta, whereby momenta expected to be in $\mathrm{GeV}$ were actually in $\mathrm{fm}^{-1}$. For the calculated two-particle azimuthal harmonics $v_{n}\left(p_{\perp}\right)$, where

$$
v_{n}^{2}\left(p_{\perp}\right)=\int \mathcal{D} \rho_{p} \mathcal{D} \rho_{t} W\left[\rho_{p}\right] W\left[\rho_{t}\right] V_{n}\left(p_{\perp}-\Delta / 2, p_{\perp}+\Delta / 2\right) V_{n}^{\star}\left(p_{\perp}^{\mathrm{ref}, \min }, p_{\perp}^{\mathrm{ref}, \max }\right),
$$

with

$$
V_{n}\left(p_{1}, p_{2}\right)=\frac{\int_{p_{1}}^{p_{2}} k_{\perp} d k_{\perp} \frac{d \phi}{2 \pi} e^{i n \phi} \frac{d N\left(\mathbf{k}_{\perp}\right)}{d^{2} k d y}\left[\rho_{p}, \rho_{t}\right]}{\int_{p_{1}}^{p_{2}} k_{\perp} d k_{\perp} \frac{d \phi}{2 \pi} \frac{d N\left(\mathbf{k}_{\perp}\right)}{d^{2} k d y}\left[\rho_{p}, \rho_{t}\right]},
$$

this affects both the measured gluon momenta $\left(p_{\perp}\right)$ as well as the momentum of the reference gluons ( $\left.p_{\perp}^{\text {ref }}\right)$. Additionally, the presented results did not correctly take into account the reference bin; instead results for $p_{\perp}^{\text {ref }}=p_{\perp}$ were shown. However we have checked that for a reasonable reference bin of $0.5<p_{\perp}^{\text {ref }}<3.0$ in the uncorrected units the previously observed ordering $v_{2}\left({ }^{3} \mathrm{He}>d>p\right)$ is preserved.

Corrected results for $v_{n}\left(p_{\perp}\right)$ using Eq. (1) are presented in Figs. 1 and 2. Here we use the same parameters utilized in our Letter with no further tuning; results are shown for $p_{\perp}^{\text {ref }}=0.5-3 \mathrm{GeV}$. In comparison to Fig. 3 of our Letter, it is clear that in Fig. 1 the previously observed hierarchy for $v_{2}\left({ }^{3} \mathrm{He}>d>p\right)$ is now reversed; i.e., we now find $v_{2}\left(p>d>{ }^{3} \mathrm{He}\right)$. Additionally, we observe that the magnitude of $v_{2}$ is significantly lower than that presented previously, and is in

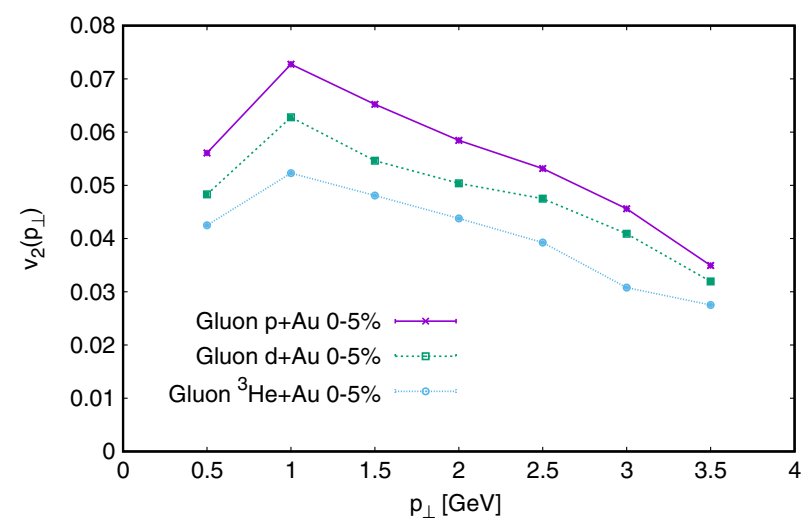

FIG. 1. Hierarchy of anisotropies $v_{2}\left(p_{\perp}\right)$ of gluons produced in the $0 \%-5 \%$ centrality class of light-heavy ion collisions computed in the dilute-dense CGC framework.

Published by the American Physical Society under the terms of the Creative Commons Attribution 4.0 International license. Further distribution of this work must maintain attribution to the author(s) and the published articles title, journal citation, and DOI. 


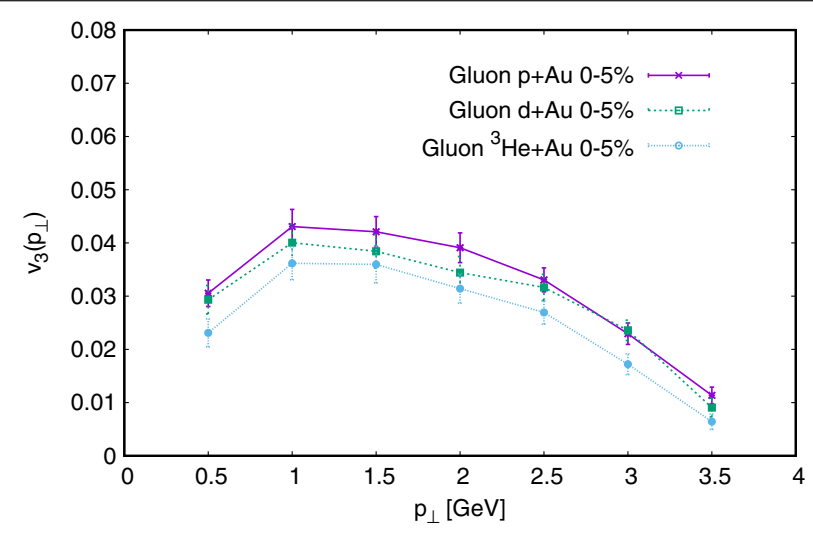

FIG. 2. Hierarchy of anisotropies $v_{3}\left(p_{\perp}\right)$ of gluons produced in the $0 \%-5 \%$ centrality class of light-heavy ion collisions computed in the dilute-dense CGC framework.

disagreement with the published experimental data from PHENIX [1]. In Fig. 2, we show our revised results for $v_{3}$. Again, we observe that the hierarchy in the magnitudes of $v_{3}$ is reversed for the three systems relative to [1].

In summary, due to the numerical error specified, the hierarchy of $v_{2,3}$ seen in the PHENIX data is not seen in our model. It cannot therefore provide a viable description of the data.

The code used to produce these results is available by request to the corresponding author.

*Corresponding author.

mark.f.mace@jyu.fi

[1] C. Aidala et al. (PHENIX Collaboration), Creation of quark-gluon plasma droplets with three distinct geometries, Nat. Phys. 15, 214 (2019). 